\title{
Changes in patterns of uveitis at a tertiary referral center in Northern Italy: analysis of 990 consecutive cases
}

\author{
Cimino Luca $(1) \cdot$ Aldigeri Raffaella $\cdot$ Marchi Sylvia $\cdot$ Mastrofilippo Valentina $\cdot$ Viscogliosi Fabiana \\ Coassin Marco • Soldani Annamaria - Savoldi Luisa • De Fanti Alessandro • Belloni Lucia • \\ Zerbini Alessandro $\cdot$ Parmeggiani Maria $\cdot$ Chersich Matthew $\cdot$ Soriano Alessandra $\cdot$ Salvarani Carlo \\ Fontana Luigi
}

Received: 9 November 2016/Accepted: 27 December 2016/Published online: 9 January 2017

(C) The Author(s) 2017. This article is published with open access at Springerlink.com

\begin{abstract}
Purpose The role of uveitis, an uncommon ocular disease, is often neglected in research and treatment of autoimmune conditions. The study described the spectrum of uveitis at a referral center in North Italy, and compared that to a previously published series of patients. Methods We reviewed all patients with uveitis diagnosed from 2013 to 2015 at the Immunology Eye Unit, Arcispedale S. M. Nuova-IRCCS, Reggio
\end{abstract}

C. Luca $(\bowtie) \cdot$ M. Sylvia · M. Valentina .

V. Fabiana $\cdot$ S. Annamaria

Immunology Eye Unit, Eye Department, Arcispedale S.

M. Nuova-IRCCS, Reggio Emilia, Italy

e-mail: cimino.luca@asmn.re.it;

1.cimino64@gmail.com

\section{A. Raffaella}

Department of Clinical and Experimental Medicine, University of Parma, Parma, Italy

e-mail: raffaella.aldigeri@unipr.it

M. Sylvia - M. Valentina - C. Marco .

S. Annamaria · F. Luigi

Eye Department, Arcispedale S. M. Nuova-IRCCS,

Reggio Emilia, Italy

e-mail: fontana.luigi@asmn.re.it

\section{S. Luisa}

Scientific Directorate, Arcispedale S.M. Nuova-IRCCS, Reggio Emilia, Italy

e-mail: savoldi.luisa@asmn.re.it
Emilia, Italy. We examined patient characteristics, disease spectrum, and etiologies.

Results In total, 990 cases of uveitis were identified, who were mostly female $(59 \%)$ with a median age at presentation of 44 years (interquartile range $=29-57$ ). Anterior uveitis was most frequent (53.5\%), followed by panuveitis (22.8\%), posterior (16.2\%), and intermediate uveitis (5.5\%). Anterior herpetic uveitis (15.6\%), Fuchs uveitis $(9.7 \%)$, and HLA-B27 positive anterior uveitis (7.7\%) were the most common specific diagnoses.

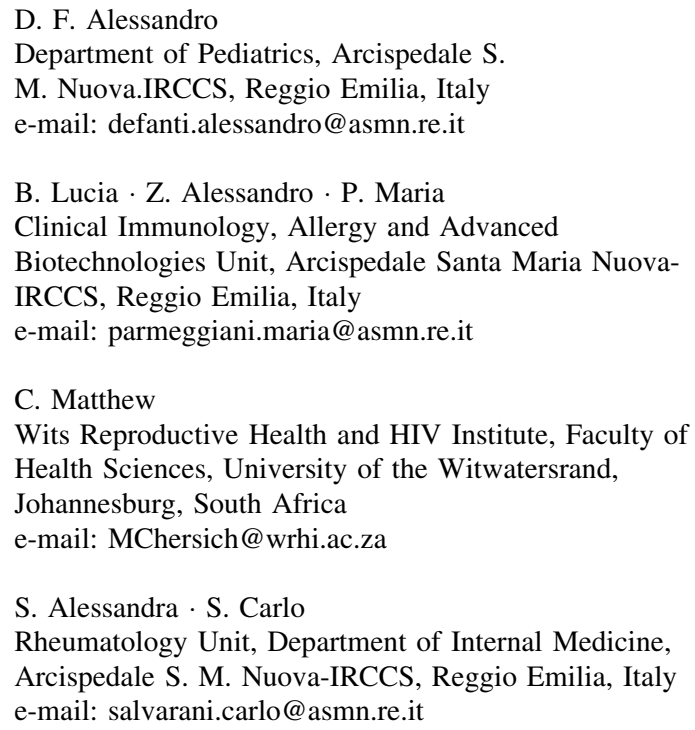


Compared with the previous series, we observed an increased incidence of uveitis, and a different pattern of diagnoses. Rates of herpetic, HLA-B27 positive uveitis, and presumed ocular tuberculosis were higher, but Fuchs uveitis was less frequent.

Conclusions The pattern of uveitis appears to be changing, very likely due to population-level increases in infectious diseases, to the availability of new diagnostic tests and to the interdisciplinary approach used in patient diagnosis.

Keywords Italy · Epidemiology · Infection · Interdisciplinary approach $\cdot$ Systemic disease $\cdot$ Uveitis

\section{Introduction}

Uveitis represents a heterogeneous group of intraocular diseases, encompassing a range of inflammatory eye conditions affecting not only the uvea, but also the retina, the optic nerve, and the vitreous. Although a rare disease, its prevalence is rising [1-3]. Uveitis can vary substantially in terms of its clinical course, management, and prognosis. While certain types of uveitis require no treatment, have a self-limited course, and a favorable prognosis, other forms carry a considerable risk of vision loss. Uveitis can be limited to the eye or associated to systemic diseases. Conditions can be idiopathic, autoimmune, or caused by many different infectious agents. Since uveitis cases are often not clinically recognized, the diagnostic delay may increase the risk of irreversible sightthreatening complications. Also, as genetic, ethnic, geographic, socioeconomic, nutritional, and environmental factors influence the pathogenesis of uveitis, it is important to assess the patterns of the condition in different geographic regions over time [3, 4]. Drawing on a computerized register of all cases of uveitis at a tertiary-level referral center in Northern Italy, we analyzed the spectrum of uveitis patients from November 2013 to October 2015 and compared it to our previous published series [5].

\section{Materials and methods}

In November 2013, a database was developed to systematically collect data on uveitis patients referred to our Immunology Eye Unit in Reggio Emilia, Italy. We here review the database entries of patients with uveitis referred to our unit over 24 months. Human Immunodeficiency Virus (HIV) positive and masquerade uveitis patients were excluded. All patients gave written informed consent for analysis of their data, and the study was approved by the Arcispedale S. Maria Nuova-IRCCS Institutional Review Board.

Demographic data, medical history, ocular symptoms and signs, special investigations and therapy of patients were analyzed. At presentation, all patients underwent the following: best-corrected Snellen visual acuity, applanation tonometry, slit-lamp evaluation of the anterior segment, and fundus examination. When appropriate, fluorescein angiography, indocyanine green angiography, optical coherence tomography, ocular ultrasonography, and visual field testing were performed. Furthermore, when required, patients underwent complete diagnostic work-up, including laboratory tests and medical evaluation. The selection of diagnostic work-up was based on the positive predictive value of the laboratory tests. For example, as HLA-B27-positive anterior uveitis does not have a granulomatous component, in patients who are HLA-B27 positive and have anterior granulomatous uveitis, two distinct pathologies were considered (for example, herpetic uveitis with concurrent HLAB27 positivity).

The anatomical classification and final diagnosis were based on the criteria of the International Uveitis Study Group (IUSG) and the Standardization of Uveitis Nomenclature (SUN) working group [6, 7]. Furthermore, granulomatous and non-granulomatous uveitis were categorized from a clinical (and not histological) point of view, a useful distinction in clinical practice, as some uveitis conditions are always non-granulomatous, such as HLA-B27 or Behçetrelated uveitis. The clinical aspects of non-granulomatous uveitis are the presence of fine endothelial keratic precipitates (dots), in the absence of iris nodules and/or choroidal granulomas, and granulomatous if larger ("mutton fat") keratic precipitates, Koeppe and/or Busacca nodules and/or choroidal granulomas [8]. Patients were referred to relevant specialists if systemic disease was suspected. Criteria for specific diagnosis included a defined etiology, typical clinical appearance, and history or classification based on pathological or diagnostic laboratory parameters. 
Here, we briefly illustrate our approach for making specific diagnoses and, when necessary, interdisciplinary work-up.

\section{HLA-B27-positive anterior uveitis}

Patients with acute anterior uveitis, always clinically non-granulomatous, and typed for the presence of the HLA-B27, regardless of the presence of systemic disease.

Anterior herpetic uveitis

Intraocular inflammation that is usually acute, unilateral, and granulomatous with posterior synechiae and sectoral iris atrophy [5]. In selected cases, where clinical presentation is atypical and/or recurrent despite antiviral therapy, we performed both the serological profile of the suspected microorganism, Herpes Virus-1 (HSV-1), Herpes Virus-2 (HSV-2), Varicella Zoster Virus (VZV), and Real-Time Polymerase Chain Reaction (RT-PCR) on the aqueous tap $[9,10]$. In case of clinical suspicion, but negative result of RT-PCR, antibody index coefficient (AIC) (Improved Goldmann-Witmer index) assessment is performed $[9,11,12]$.

\section{Anterior cytomegalovirus (CMV) uveitis}

In the case of granulomatous anterior uveitis, unilateral, recurrent episodic uveitis, without sectorial iris atrophy, with raised intraocular pressure, open angle, immunocompetent patients, after the assessment of seropositivity for CMV infection, we confirm the diagnosis with an aqueous tap for RT-PCR analysis, and Antibody Index testing in case of negative results of RT-PCR [11-13].

Fuchs uveitis

It is mostly unilateral granulomatous uveitis involving the anterior segment and the vitreous body. The main clinical signs are characteristic sparsely distributed stellate granulomatous keratic precipitates, iris stroma atrophic changes, the absence of synechiae, anterior vitritis, and the absence of cystoid macular oedema [5].
Toxoplasmosis "typical retinochoroiditis"

The diagnosis is based on a compatible fundus lesion and positive serology for toxoplasma antibodies. A solitary inflammatory focus near an old pigmented scar is typical [5]. If the diagnosis is uncertain in patients seropositive for toxoplasmosis, an aqueous sample is used to confirm the diagnosis with RT-PCR $[7,12]$.

Acute retinal necrosis (ARN)

The diagnosis was based on the criteria of American Uveitis Society [8].

Sarcoidosis

The presumed diagnosis is made on consistent clinical findings with two of the following three diagnostic criteria: elevated angiotensin converting enzyme or lysozyme, cutaneous anergy, and/or with positive chest tomography [14]. We prefer to obtain diagnostic confirmation directly by a positive mediastinoscopic lymphonodular biopsy rather than by interlocutory tests (such as bronchoalveolar lavage) or trans-bronchiolar biopsy (sometimes difficult to obtain).

Pars planitis

It commonly indicates a subset of intermediate uveitis where there is snowbank or snowball formation occurring in the absence of an associated infection or systemic disease (that is, "idiopathic") [7].

Behçet's disease (BD)

We used the International Study Group (ISG) criteria for BD [15], which includes the presence of recurrent oral ulcers plus two of the following: (a) recurrent genital ulcers, (b) eye lesions, (c) skin lesions, and (d) positive pathergy test.

Presumed tuberculous (TB) uveitis

We define any kind of uveitis compatible with a tuberculous etiology with Quantiferon Gold TB positivity [16] or Mantoux tuberculin skin test (2U) $>15 \mathrm{~mm}$ diameter of induration at $48-72 \mathrm{~h}$, with or without abnormalities on chest X-ray, exclusion of 
other possible causes of uveitis, and the response to anti-tuberculosis treatment [5].

\section{Primary Inflammatory Choriocapillaropathies (PICCP)}

The common denominator in PICCP is choriocapillaris non-perfusion and secondary ischaemia of the outer retina. Autofluorescence (FAF), Fluoroangiography (FA), and Indocyanine green angiography (ICG) are fundamental diagnostic tests for detecting choriocapillaris non-perfusion [17]. The clinical differences between the PICCP could possibly be explained by the level and the severity of the inflammation of the choriocapillaris circulation. Before the diagnosis of PICCP is made, an infectious cause, a neoplastic process, or a systemic vasculitis must be ruled out. The classification of PICCP into any of the known and well-defined entities is useful in predicting evolution and defining therapy [5, 18]: multiple evanescent white dot syndrome (MEWDS), acute posterior multifocal placoid pigment epitheliopathy (APMPPE), multifocal choroiditis, and serpiginous choroiditis.
Vogt-Koyanagi-Harada (VKH) disease

It is a multisystem disease. Chronic, bilateral, granulomatous panuveitis are associated with the central nervous system, auditory and integumentary manifestations. Its hallmark is bilateral multifocal exudative retinal detachments, hyperemia, and edema of the optic disk. We include lumbar puncture when necessary at the onset of the disease, especially in patients without systemic steroid therapy showing cerebrospinal fluid (CSF) pleocytosis [19, 20].

The term idiopathic is used whenever the intraocular inflammation cannot be attributed to a specific diagnosis.

Descriptive data are presented as mean and standard deviation (SD), or median and interquartile range (IQR), where appropriate. Categorical variables are expressed as frequency and percentages. To assess differences among groups, Student's $t$ test and ANOVA were used for continuous data, and Chisquare tests were conducted for categorical variables. Statistical analysis was performed using SPSS (IBM SPSS statistics v.22).

Table 1 Demographic and clinical characteristics of patients, by time period and anatomical site

\begin{tabular}{|c|c|c|c|c|c|c|c|}
\hline \multirow[t]{2}{*}{ Variable } & \multirow{2}{*}{$\begin{array}{l}\text { Cases 2002-2008 } \\
(N=1064)\end{array}$} & \multicolumn{5}{|c|}{ Cases 2013-2015 } & \multirow[t]{2}{*}{$P$} \\
\hline & & $\begin{array}{l}\text { Total cases } \\
N=990\end{array}$ & $\begin{array}{l}\text { Anterior } \\
N=530\end{array}$ & $\begin{array}{l}\text { Intermediate } \\
N=74\end{array}$ & $\begin{array}{l}\text { Panuveitis } \\
N=226\end{array}$ & $\begin{array}{l}\text { Posterior } \\
N=160\end{array}$ & \\
\hline $\begin{array}{l}\text { Median age at } \\
\text { presentation years } \\
\text { (IQR) }\end{array}$ & $40(26-55)$ & $44(29-57)^{*}$ & $45(32-59)$ & $26(15-46)$ & $45(30-58)$ & $42(29-55)$ & $<0.001$ \\
\hline Gender & & & & & & & 0.300 \\
\hline Male & $481(45)$ & $403(41)^{*}$ & $204(38)$ & $36(49)$ & $93(41)$ & $70(44)$ & \\
\hline Female & $583(55)$ & 587 (59) & $326(62)$ & $38(51)$ & $133(59)$ & $90(56)$ & \\
\hline Site & & & & & & & $<0.001$ \\
\hline Unilateral & 825 (78) & $499(50)^{*}$ & 364 (69) & $13(18)$ & $57(25)$ & $65(41)$ & \\
\hline Bilateral & $239(22)$ & $491(50)$ & $166(31)$ & $61(82)$ & $169(75)$ & $95(59)$ & \\
\hline Granulomatous & & & & & & & $<0.001$ \\
\hline Yes & $553(52)$ & $545(55)$ & $312(59)$ & $13(18)$ & $147(65)$ & $73(46)$ & \\
\hline No & $511(48)$ & $445(45)$ & $218(41)$ & $61(82)$ & $79(35)$ & $87(54)$ & \\
\hline Non-Italian ethnicity & $31(3)$ & $106(11)^{*}$ & $43(8)$ & $6(8)$ & 44 (19) & $13(8)$ & $<0.001$ \\
\hline Has a specific diagnosis & $787(74)$ & 759 (77) & $404(76)$ & $45(61)$ & $177(78)$ & $133(84)$ & 0.002 \\
\hline Infectious etiology & $257(24)$ & $301(30)^{*}$ & $173(43)$ & $3(7)$ & $68(38)$ & $57(43)$ & $<0.001$ \\
\hline Systemic disease & na & $259(26)$ & $114(21)$ & 12 (16) & $110(49)$ & $23(14)$ & $<0.001$ \\
\hline
\end{tabular}

* Indicates $P$ value $<0.05$ for comparison between previous cases and current. Data are reported as median (interquartile range) or $n(\%)$ 


\section{Results}

Nine-hundred and ninety consecutive patients with uveitis (1481 eyes) diagnosed from November 1, 2013 to October 31, 2015 were included. The majority were female $(n=587,59 \%$; Table 1$)$. There were 120 $(12 \%)$ cases among children $(\leq 18$ years), while 713 (72\%) predominated in those aged 19-64, and 157 $(16 \%)$ in those aged above $65(p<0.001)$. Age at onset of uveitis was a median 39 (IQR 24-54), while age at presentation at our clinic was a median 44 years (IQR 29-57). The majority was of Italian ethnicity $(884,89 \%)$. Of these, $57 \%$ were resident in Emilia Romagna region, $79 \%$ in other parts of Northern Italy, and $21 \%$ in Central-Southern Italy.

Anterior uveitis was the most frequent $(53.5 \%$ of cases), followed by panuveitis $(22.8 \%)$, posterior $(16.2 \%)$, and intermediate uveitis $(7.5 \%)$. There were considerable differences in disease location of uveitis in terms of age, but not of gender (Table 1). Unilateral involvement was significantly higher in anterior uveitis $(69 \%)$ than in other anatomical sites. Granulomatous forms were especially prevalent in anterior

Table 2 Frequency of specific diagnoses ( $n, \%)$

\begin{tabular}{lrr}
\hline Specific diagnosis & \multicolumn{1}{c}{$N$} & $\%$ \\
\hline Anterior herpetic uveitis & 154 & 15.6 \\
Fuchs uveitis & 96 & 9.7 \\
HLA-B27+anterior uveitis & 76 & 7.7 \\
Presumed ocular tuberculosis & 56 & 5.7 \\
Behçet & 48 & 4.8 \\
JIA & 47 & 4.7 \\
Toxoplasmosis & 47 & 4.7 \\
Presumed sarcoidosis & 43 & 4.3 \\
Vogt-Koyanagi-Harada & 41 & 4.1 \\
PICCP & 40 & 4.0 \\
Pars planitis & 33 & 3.3 \\
Anterior Cytomegalovirus uveitis & 17 & 1.7 \\
ARN & 9 & 0.9 \\
Multiple sclerosis & 9 & 0.9 \\
Birdshot retinochoroiditis & 8 & 0.8 \\
Syphilis & 8 & 0.8 \\
Serpiginous tuberculosis & 5 & 0.5 \\
Sympathetic ophthalmia & 5 & 0.5 \\
Tinu & 5 & 0.5 \\
Candida & 5 & 0.3 \\
Others & 3 & 0.2 \\
\hline
\end{tabular}

and panuveitis (59 and 65\%, respectively). Panuveitis was the more frequent among patients of non-Italian ethnicity.

An etiological diagnosis of uveitis was established for $77 \%$ of patients, with the remainder classified as having idiopathic disease (Table 2). Among specific diagnoses, the most frequent were anterior herpetic uveitis $(15.6 \%)$, followed by Fuchs' uveitis $(9.7 \%)$, HLA-B27-positive anterior uveitis (7.8\%), and presumed tuberculous uveitis (5.7\%, Fig. 1) The most frequent specific diagnoses in those aged below 18 years were Juvenile Idiopathic Arthritis (JIA) (31.9\%), pars planitis (14.7\%), followed by VKH and anterior herpetic uveitis $(6.0 \%)$. Among adults (19-64 years), the most common specific diagnosis was anterior herpetic uveitis $(14.0 \%)$, followed by Fuchs uveitis (12.3\%) and HLA B27-positive anterior uveitis $(9.8 \%)$. In older subjects ( $\geq 65$ years), the most prevalent entities were anterior herpetic uveitis (33.3\%), presumed tuberculous uveitis $(14.7 \%)$, and presumed sarcoidosis (9.8\%) (Fig. 2).

Women were considerably more likely to have had a specific diagnosis $(p=0.004)$, except for Behçet disease (56\% males vs $44 \%$ females), CMV (71\% males vs $29 \%$ females), and syphilis (75\% males vs $35 \%$ females).

Infectious uveitis was diagnosed in 301 subjects (30\% of total cases), most due to HSV (51\%), followed by tuberculosis (19\%), and toxoplasmosis $(16 \%)$ (Table 1). Infectious uveitis was more frequent in males $(34 \%)$ than females $(28 \%)$ and in subjects over age 65 years (54\%), compared to $12 \%$ in children and $28 \%$ in middle-age subjects $(p<0.001)$. In 150 $(50 \%)$ patients with presumed infectious uveitis, ocular fluid biopsy was performed in order to confirm the clinical suspicion. Forty-seven samples (31.3\%) tested positive for the following infectious agents: 18 CMV, $12 \mathrm{HSV}, 11$ Toxoplasma, $5 \mathrm{VZV}$, and 1 Listeria. Sixteen patients positive for CMV and 9 positive for HSV-1 presented with hypertensive anterior granulomatous uveitis.

In a quarter of cases, uveitis was associated with systemic diseases (259/990), with levels higher among females (29\%) than males $(22 \%, p=0.016)$ and in the younger age subjects $(44 \%)$ than those aged above 18 years $(25 \%, p<0.0001)$. According to anatomical classification, in those with systemic disease, panuveitis was the most prevalent $(49 \%)$, followed by anterior $(21 \%)$, intermediate $(19 \%)$, and posterior 


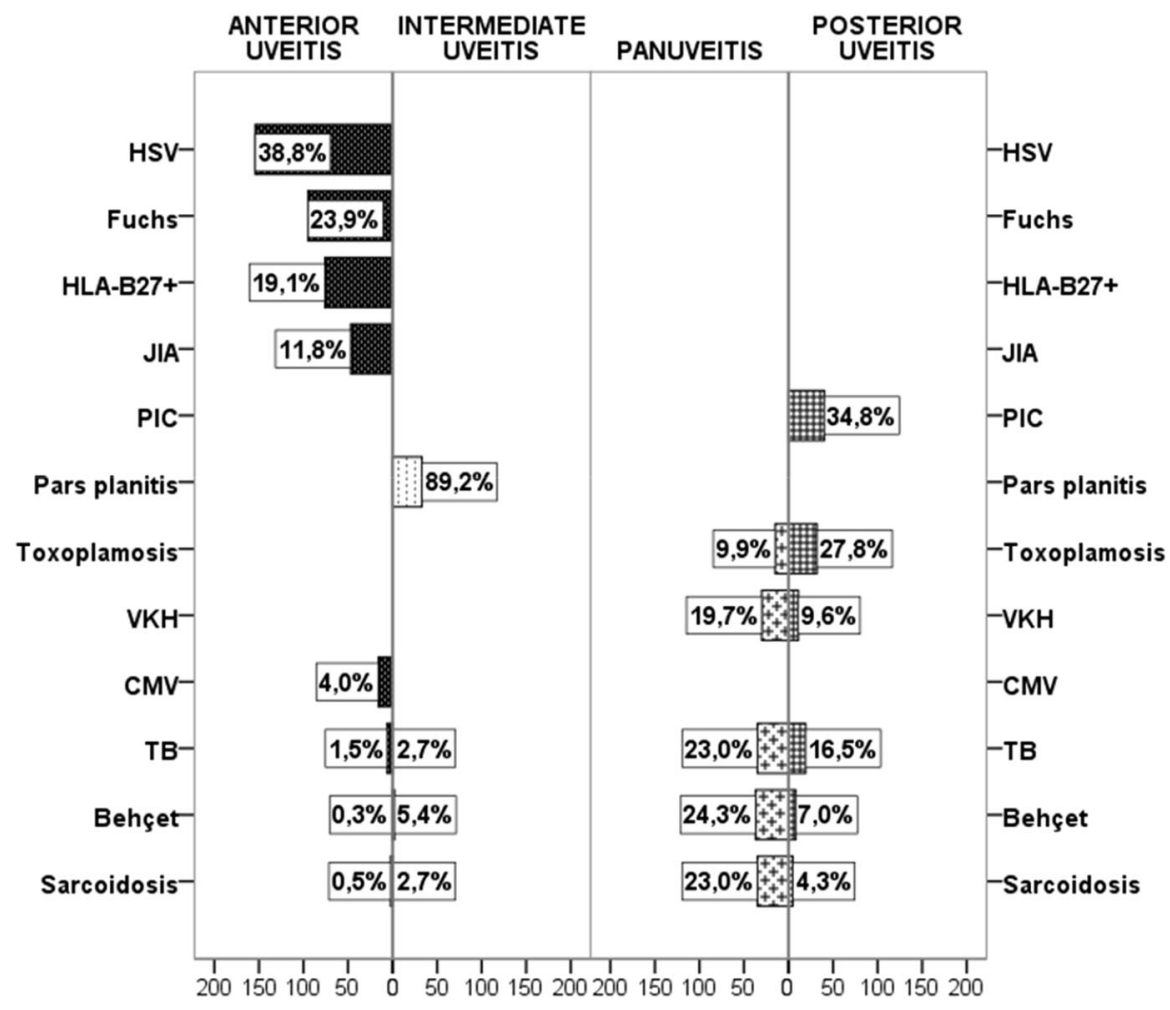

Fig. 1 Distribution of the most frequent specific diagnoses according to anatomical classification

uveitis $(15 \%, p<0.0001)$. The most common specific diagnoses in patients with systemic diseases were JIA and Behçet's ( $n=47,18.5 \%)$, followed by presumed sarcoidosis $(n=43,17 \%)$, VKH, and HLA-B27positive anterior uveitis $(n=41,16.1 \%)$.

With regard to treatment, $277(28 \%)$ patients were on systemic therapy, with steroids alone $(22.5 \%)$, or steroids and either immunomodulatory agents (IMiDs) $(18.2 \%)$ or biologics $(4.0 \%)$, or with all three of these drug groups (2.9\%). Fifty-three patients $(19.3 \%)$ had been treated with biologics and immunomodulatory agents alone (Fig. 3). Overall, 549 (56\%) patients developed at least one complication. Cataract was the most common, occurring in $19 \%$ of cases, followed by posterior synechiae (14\%), macular edema (13\%), and glaucoma (11\%).

\section{Discussion}

This is only the fourth epidemiological study on uveitis in Italy [5, 21, 22], with the most recent one covering the period 2002-2008 [5]. In the same territory (533,000 inhabitants) during 24 months (2013-2015), we observed 164 new cases of uveitis, giving an estimated incidence of 15.4 cases per year per 100,000 inhabitants, higher than the value in our previous analysis in 2008 (14.6/year/100,000 people).

The distribution of cases showed the predominance of anterior uveitis, consistent with the previous studies [23-57], including our own [5]. In the last series from our site, age at presentation was significantly higher with a significant female prevalence (see Table 1). As compared to the previous series, we observed a similar frequency of anterior and intermediate uveitis (54 and $51 \%$, and 7 and $6 \%$, respectively) and a significant prevalence of panuveitis (in the latter study) respect to posterior uveitis (23 vs $20 \%, p<0.001$ ).

In this study, specific diagnoses were made in $77 \%$ of patients, higher than in other reports, [5, 23-57] which range from 42 to $75 \%$. The increase of specific diagnoses is likely the result of both an interdisciplinary approach and the introduction of new diagnostic techniques. PCR and Antibody Index tests on 


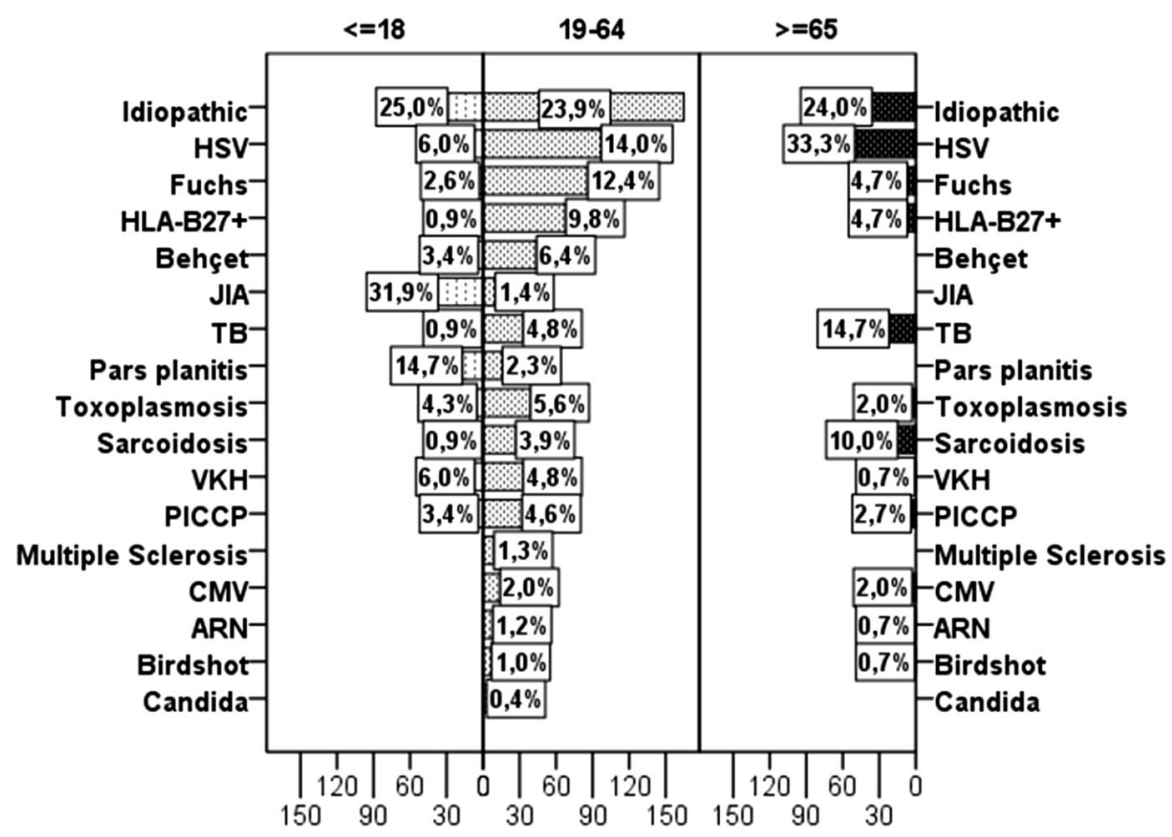

Fig. 2 Age distribution of most frequent diagnoses

eye fluids helped to confirm the diagnosis of different herpetic etiologies.

Also, the introduction of interferon gamma tests like Quantiferon helped to confirm the diagnosis of tuberculosis.

The prevalence of infectious uveitis $(30 \%)$ was significantly higher $(p=0.002$, Table 1$)$ than in our previous series (24\%): anterior herpetic uveitis, the most common specific diagnosis, increased from $9.1 \%$ in 2008 to $15.6 \%$, while no difference was detected between the proportion with tuberculosis (4.4 in 2008 to $5.7 \%$ in current series, $p=0.19$ ). Eye fluids analysis was able to confirm some specific infectious etiologies, e.g., HSV and CMV, while other diagnoses, such as Fuchs uveitis, decreased from 22.7 to $9.7 \%$ in the latter series. We observed also a lower prevalence of toxoplasmosis, from levels of $6.9 \%$ in the first analysis to $4.7 \%$ in the present analysis (Table 3 ).

Tuberculosis and syphilis accounted for $20 \%$ and almost $3 \%$ of infectious etiologies, respectively, an increase in the recent years, possibly due to the rise in immigration in Italy. The percentage of non-Italian ethnicity among patients rose from $3 \%$ in the first series to $10.7 \%$ in this report $(p<0.0001$, Table 1$)$.

The relatively high percentage of uveitis associated with systemic diseases (JIA, sarcoidosis, VKH, HLA-
B27) is consistent with what Barisani-Asenbauer et al. reported [24], underlining the need for an interdisciplinary approach to reduce the diagnostic delay, and thus ocular as well as systemic complications due to non-appropriate therapies. In our series, 44 patients presented acute anterior non-granulomatous uveitis (38/42 HLA-B27-positive anterior uveitis) associated with ankylosing spondylitis. In 35/42 of cases, the ocular diagnosis preceded the systemic one.

The leading complications observed in our study were cataract, glaucoma, and macular edema, in line with several previous reports [8]. Regarding medications, traditional immunomodulatory agents still seem to be preferred by ophthalmologists in autoimmune conditions, rather than biologics, except for HLAB27-associated uveitis, where anti-TNFs are prescribed more frequently.

Diagnostic delays are often due to difficulties in establishing the primary diagnosis of uveitis. Comparisons among surveys conducted in uveitis clinics elsewhere in the world often present great differences in the prevalence of diagnostic categories (Table 3). Such differences are probably related to genetic, geographical, social, and environmental factors. They may also be attributable to heterogeneity in the diagnostic criteria and definitions, together with the 


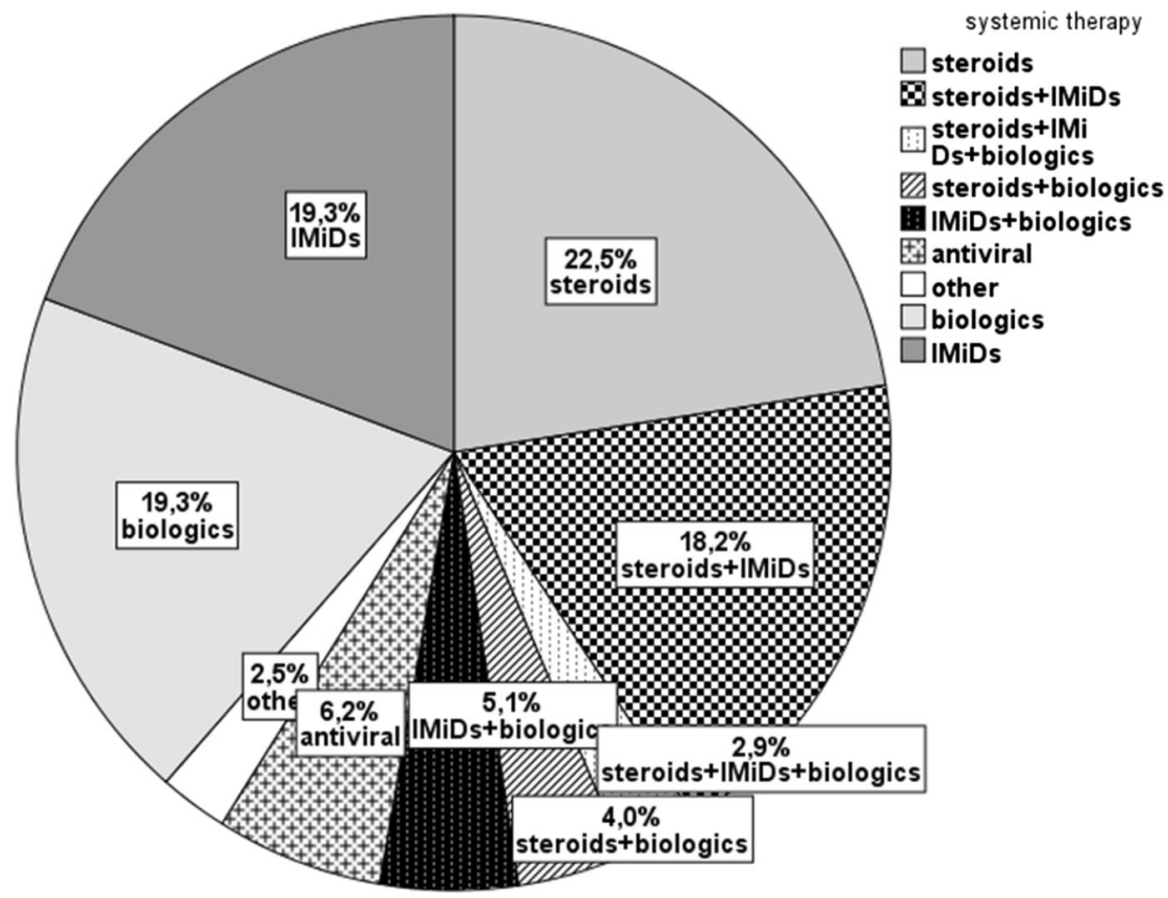

Fig. 3 Ongoing systemic therapy ( $n=277$ cases $)$

availability of different investigation techniques, as well as different interpretations of the anatomical categorization of intraocular inflammation.
In conclusion, the pattern of uveitis appears to be changing, very likely due to the spreading of infectious diseases, to the availability of new diagnostic

Table 3 Frequencies (percentages of total cases) of key uveitis diagnoses in comparison with previous studies

\begin{tabular}{|c|c|c|c|c|c|c|c|c|c|c|}
\hline Country & Author & $N ;$ Period & Fuchs & $\begin{array}{l}\text { HLA- } \\
\text { B27 }\end{array}$ & HSV & Behçet's & Toxoplasmosis & Sarcoidosis & $\mathrm{TB}$ & VKH \\
\hline \multirow[t]{4}{*}{ Italy } & Present data & $990 ; 2013-2015$ & 9.7 & 7.7 & 15.6 & 4.8 & 4.7 & 4.3 & 5.7 & 4.1 \\
\hline & Cimino $^{9}$ & $1064 ; 2002-2008$ & 22.7 & 5.3 & 9.9 & 5.3 & 6.9 & 2.2 & 4.4 & 1.9 \\
\hline & Mercanti $^{24}$ & $655 ; 1986-1993$ & 2.1 & na & 11.7 & 3.0 & 17.7 & 0.8 & 7.0 & 1.4 \\
\hline & Pivetti-Pezzi ${ }^{25}$ & $1417 ; 1986-1993$ & 8.3 & 3.4 & 5.3 & 1.3 & 6.6 & 0.2 & na & 2.0 \\
\hline UK & Jones $^{26}$ & $3000 ; 1991-2013$ & 11.5 & 4.5 & 1.9 & 2.7 & 6.9 & 9.7 & 3.3 & 0.8 \\
\hline Austria & $\begin{array}{l}\text { Barisani- } \\
\text { Asenbauer }^{27}\end{array}$ & $2619 ; 1995-2009$ & 4.6 & 19.5 & 7.1 & 1.9 & 6.6 & 3.2 & 0.8 & 0.4 \\
\hline Spain & Llorenç $^{28}$ & $1022 ; 2009-2012$ & 1.0 & 13.0 & 12.0 & 5.0 & 7.0 & 3.0 & 5.0 & 1.0 \\
\hline \multirow[t]{2}{*}{ Germany } & Grajewski $^{29}$ & $476 ; 2012-2013$ & 7.0 & 19.0 & 12.0 & 2.0 & 7.1 & 11.0 & na & 0.6 \\
\hline & $\mathrm{Jacob}^{30}$ & $1916 ; 2001-2006$ & 6.9 & 7.1 & 6.1 & 1.8 & 4.2 & 4.5 & 1.1 & 0 \\
\hline Swiss & $\operatorname{Tran}^{31}$ & $558 ; 1990-1993$ & 5.4 & 15.9 & 13.8 & 1.1 & 9.5 & 5.9 & 9.5 & 0.2 \\
\hline France & Bodaghi $^{32}$ & $927 ; 1991-1996$ & 2.5 & 4.7 & 8.6 & 6.1 & 11.9 & 6.4 & 4.1 & 2.0 \\
\hline Japan & Nakahara $^{39}$ & $695 ; 2010-2012$ & 2.1 & 3.0 & 6.0 & 5.6 & 0 & 9.4 & 1.5 & 7.9 \\
\hline Saudi Arabia & Al Dhahri ${ }^{18}$ & $642 ; 1998-2013$ & 11.3 & 9.0 & 10.0 & 8.4 & 6.9 & 4.5 & 17.8 & 19.6 \\
\hline Tunisia & Khairallah $^{47}$ & $472 ; 1992-2003$ & 3.0 & 2.8 & 11.9 & 12.3 & 10.1 & 1.7 & 1.1 & 4.4 \\
\hline Turkey & Kazokoglu $^{49}$ & $761 ; 2004$ & 5.1 & 2.4 & 2.8 & 32.1 & 4.7 & 0.9 & 0.3 & 1.1 \\
\hline US & Rodriguez $^{50}$ & $1237 ; 1982-1992$ & 2.6 & 3.8 & 7.2 & 2.5 & 4.8 & 9.6 & 0.6 & 0.9 \\
\hline
\end{tabular}


tests, such as aqueous analysis by PCR and AIC, and to more refined classification (such as the PICCP grouped together on the basis of similar etiology and particular clinical and imaging aspects). Moreover, an interdisciplinary approach that includes ophthalmologists, rheumatologists, pediatricians, and infectious disease specialists with uveitis experience may help to raise the standards of treatment, and improve prognosis and clinical course of the disease, thereby reducing the risk of complications.

Acknowledgements We thank Dr. Paola Masini, Servizio Tecnologie Informatiche e Telematiche (STIT) ASMN-IRCCS, for technical support in the database.

\section{Compliance with ethical standards}

Conflicts of interest All authors have no conflict of interest to declare.

Ethical approval All procedures performed in the studies involving human participants were in accordance with the ethical standards of the institutional and/or national research committee and with the 1964 Helsinki declaration and its later amendments or comparable ethical standards.

Informed consent Informed consent was obtained from all individual participants included in the study.

Open Access This article is distributed under the terms of the Creative Commons Attribution 4.0 International License (http:// creativecommons.org/licenses/by/4.0/), which permits unrestricted use, distribution, and reproduction in any medium, provided you give appropriate credit to the original author(s) and the source, provide a link to the Creative Commons license, and indicate if changes were made.

\section{References}

1. Henderly DE, Genstler AJ, Smith RE, Rao NA (1987) Changing patterns of uveitis. Am J Ophthalmol 103:131-136

2. Rathinam SR, Namperumalsamy P (2007) Global variation and pattern changes in epidemiology of uveitis. Indian $\mathbf{J}$ Ophthalmol 55:173-183

3. Wakefield D, Chang JH (2005) Epidemiology of uveitis. Int Ophthalmol Clin 45:1-13

4. McCannel CA, Holland GN, Helm CJ et al (1996) Causes of uveitis in the general practice of ophthalmology. UCLA Community-Based Uveitis Study Group. Am J Ophthalmol 121:35-46

5. Cimino L, Aldigeri R, Salvarani C et al (2010) The causes of uveitis in a referral centre of Northern Italy. Int Ophthalmol 30:521-529

6. Bloch-Michel E, Nussenblatt RB (1987) International uveitis study group recommendations for the evaluation of intraocular inflammatory disease. Am J Ophthalmol 103:234-235

7. Jabs DA, Nussenblatt RB, Rosenbaum JT (2005) Standardization of uveitis nomenclature for reporting clinical data. Results of the First International Workshop. Am J Ophthalmol. 140:509-516

8. Al Dhahri H, Al Rubaie K, Hemachandran S et al (2014) Patterns of uveitis in a university-based tertiary referral center in Riyadh, Saudi Arabia. Ocul Immunol Inflamm 24:1-9

9. De Groot-Mijnes JD, Rothova A, Van Loon AM et al (2006) Polymerase chain reaction and Goldmann-Witmer coefficient analysis are complimentary for the diagnosis of infectious uveitis. Am J Ophthalmol 141:313-318

10. Van der Lelij A, Ooijman FM, Kijlstra A, Rothova A (2000) Anterior uveitis with sectoral iris atrophy in the absence of keratitis: a distinct clinical entity among herpetic eye diseases. Ophthalmology 107:1164-1170

11. Quentin CD, Reiber H (2004) Fuchs heterochromic cyclitis: Rubella virus antibodies and genome in aqueous humor. Am J Ophthalmol 138:46-54

12. Cimino L, Aldigeri R, Parmeggiani M et al (2013) Searching for viral antibodies and genome in intraocular fluids of patients with Fuchs uveitis and non-infectious uveitis. Graefes Arch Clin Exp Ophthalmol 251:1607-1612

13. de Schryver I, Rozenberg F, Cassoux N et al (2006) Diagnosis and treatment of cytomegalovirus iridocyclitis without retinal necrosis. Br J Ophthalmol 90:852-855

14. Herbort CP, Mochizuki M, Rao NA, and the members of the Scientific Committee of the First International Workshop on Ocular Sarcoidosis IWOS (2009) International criteria for the diagnosis of ocular sarcoidosis: results of the first International Workshop on Ocular Sarcoidosis (IWOS). Ocul Immunol Inflamm 17:160-169

15. International Study Group for Behçet's Disease (1990) Evaluation of diagnostic "classification" criteria in Behcet's disease: toward internationally agreed criteria. Lancet 335:1078-1080

16. Gupta V, Gupta A, Rao NA (2007) Intraocular tuberculosisan update. Surv Ophthalmol 52:561-587

17. Mantovani A, Giani A, Herbort CP, Staurenghi G (2016) Interpretation of fundus autofluorescence changes in choriocapillaritis: a multi-modality imaging study. Graefes Arch Clin Exp Ophthalmol 254:1473-1479

18. Chang J, Wakefield D (2002) Uveitis a global perspective. Ocul Immunol Inflamm 10:263-279

19. Moorthy RS, Inomata H, Rao NA (1995) Complications and prognostic factors in Vogt-Koyanagi-Harada disease. Surv Ophthalmol 39:265-292

20. Read RW, Rechodouni A, Butani N et al (2001) Complications and prognostic factors in Vogt-Koyanagi-Harada disease. Am J Ophthalmol 131:599-606

21. Mercanti A, Parolini B, Bonora A et al (2001) Epidemiology of endogenous uveitis in northeastern Italy. Analysis of 655 new cases. Acta Ophthalmol Scand 79:64-68

22. Pivetti-Pezzi P, Accorinti M, La Cava M et al (1996) Endogenous uveitis: an analysis of 1,417 cases. Ophthalmologica 10:234-238

23. Jones NP (2015) The manchester uveitis clinic: the first 3000 patients-epidemiology and casemix. Ocul Immunol Inflamm 23:118-126 
24. Barisani-Asenbauer T, Maca SM, Mejdoubi L et al (2012) Uveitis-a rare disease often associated with systemic diseases and infections: a systemic review of 2619 patients. Orphanet J Rare Dis 7:57-64

25. Llorenç V, Mesquida M, Sainz de la Maza M et al (2015) Epidemiology of uveitis in a Western urban multiethnic population. The challenge of globalization. Acta Ophthalmol 93:561-567

26. Grajewski RS, Caramoy A, Frank KF et al (2015) Spectrum of uveitis in a German tertiary center: review of 474 consecutive patients. Ocul Immunol Inflamm 23:346-352

27. Jakob E, Reuland MS, Mackensen F et al (2009) Uveitis subtypes in a German interdisciplinary uveitis centeranalysis of 1916 patients. J Rheumatol 36:127-136

28. Tran VT, Auer C, Gueex-Crosier Y et al (1994) Epidemiology of uveitis in Switzerland. Ocul Immunol Inflamm 2:169-176

29. Bodaghi B, Cassoux N, Wechsler B et al (2001) Chronic severe uveitis: etiology and visual outcome in 927 patients from a single center. Medicine (Baltim) 80:263-270

30. Liberman P, Gauro F, Berger O, Urzua CA (2015) Causes of uveitis in a tertiary center in Chile: a cross-sectional retrospective review. Ocul Immunol Inflamm 23:339-345

31. Singh R, Gupta V, Gupta A (2004) Pattern of uveitis in a referral eye clinic in north India. Indian $\mathrm{J}$ Ophthalmol 52:121-125

32. Soheilian M, Heidari K, Yazdani S et al (2004) Patterns of uveitis in a tertiary eye care center in Iran. Ocul Immunol Inflamm 12:297-310

33. Kianersi F, Mohammadi Z, Ghanbari H et al (2015) Clinical patterns of uveitis in an Iranian tertiary eye-care center. Ocul Immunol Inflamm 23:278-282

34. Kitamei H, Kitaichi N, Namba K et al (2009) Clinical features of intraocular inflammation in Hokkaido, Japan. Acta Ophthalmol 87:424-428

35. Keino H, Nakashima C, Watanabe T et al (2009) Frequency and clinical features of intraocular inflammation in Tokyo. Clin Experiment Ophthalmol 37:595-601

36. Nakahara H, Kaburaki T, Takamoto M et al (2015) Statistical analyses of endogenous uveitis patients (2007-2009) in central Tokyo area and comparison with previous studies (1963-2006). Ocul Immunol Inflamm 23:291-296

37. Abdulaal M, Antonios R, Barikian A et al (2015) Etiology and clinical features of ocular inflammatory diseases in a tertiary center in Lebanon. Ocul Immunol Inflamm 23:271-277

38. Hamade IH, Elkum N, Tabbara KF (2009) Causes of uveitis at a referral center in Saudi Arabia. Ocul Immunol Inflamm 17:11-16

39. Al-Mezaine HS, Kangave D, Abu El-Asrar AM (2010) Patterns of uveitis in patients admitted to a university hospital in Riyadh, Saudi Arabia. Ocul Immunol Inflamm 18:424-431

40. Mi H, Ho SL, Lim WK, Wong EP, Teoh SC (2015) Trends in patterns of posterior uveitis and panuveitis in a tertiary institution in Singapore. Ocul Immunol Inflamm 23:329-338

41. Pathanapitoon K, Kunavisarut P, Ausayakhun S et al (2008) Uveitis in a tertiary ophthalmology centre in Thailand. Br J Ophthalmol 92:474-478

42. Sittivarakul W, Bhurayanontachai P, Ratanasukon M (2013) Pattern of uveitis in a university-based referral center in southern Thailand. Ocul Immunol Inflamm 21:53-60

43. Silpa-Archa S, Noonpradej S, Amphornphruet A (2015) Pattern of uveitis in a referral ophthalmology center in the central district of Thailand. Ocul Immunol Inflamm 23:320-328

44. Khairallah M, Yahia SB, Ladjimi A et al (2007) Pattern of uveitis in a referral centre in Tunisia, North Africa. Eye (Lond) 21:33-39

45. Nalcacioglu-Yuksekkaya P, Ozdal PC, Yazici A, Tirhis H (2015) Clinical and demographic characteristics of patients with uveitis starting later in life. Ocul Immunol Inflamm 23:304-310

46. Kazokoglu H, Onal S, Tugal-Tutkun I et al (2008) Demographic and clinical features of uveitis in tertiary centers in Turkey. Ophthalmic Epidemiol 15:285-293

47. Rodriguez A, Calonge M, Pedroza-Seres M et al (1996) Referral patterns of uveitis in a tertiary eye care center. Arch Ophthalmol 114:593-599

48. Bajwa A, Osmanzada D, Osmanzada S et al (2015) Epidemiology of uveitis in the mid-Atlantic United States. Clin Ophthalmol 20:889-901

49. Karaconji T, Maconochie Z, McCluskey P (2013) Acute anterior uveitis in Sydney. Ocul Immunol Inflamm 21:108-114

50. Wakefield D, Dunlop I, McCluskey PJ, Penny R (1986) Uveitis: aetiology and disease associations in an Australian population. Aust N Z J Ophthalmol 14:181-187

51. Chao JR, Khurana RN, Fawzi AA, Reddy HS, Rao NA (2006) Syphilis: reemergence of an old adversary. Ophthalmology 113:2074-2079

52. Sengun A, Karadag R, Karakurt A et al (2005) Causes of uveitis in a referral hospital in Ankara, Turkey. Ocul Immunol Inflamm 13:45-50

53. Miyanaga M, Shimizu K, Kawaguchi T et al (2009) A clinical survey of uveitis in HTLV-1 endemic region. Ocul Immunol Inflamm 17:335-341

54. Bodaghi B, Wechsler B, Du-Boutin LT et al (2003) Chronic severe uveitis: classification, search for etiology and therapeutic approach. Rev Med Interne 24:794-802

55. Miserocchi E, Fogliato G, Modorati G, Bandello F (2013) Review on the worldwide epidemiology of uveitis. Eur $\mathbf{J}$ Ophthalmol 23:705-717

56. Merrill PT, Kim J, Cox TA et al (1997) Uveitis in Southeastern United States. Curr Eye Res 16:865-874

57. Oruc S, Kaplan AD, Galen M, Kaplan HJ (2003) Uveitis referral pattern in a Midwest University Eye Center. Ocul Immunol Inflamm 11:287-298 